\title{
Menopausia: Panorama actual de manejo
}

\author{
Luis ERnesto Pérez A. MD. Ginecólogo Obstetra ${ }^{* *}$ e Ingrid C. Rojas MD. ${ }^{2}$ \\ ${ }^{1}$ Profesor Titular, Facultad de Medicina, Universidad Militar Nueva Granada, Hospital Militar, Bogotá, DC, Colombia. \\ ${ }^{2}$ Médico general, Universidad Militar Nueva Granada, Bogotá, DC, Colombia.
}

\begin{abstract}
Resumen
Aún existe, desde 2002, una marcada disminución en el uso de terapia hormonal clásica (TH), para el manejo de la menopausia, debido a los riesgos determinados en el estudio Iniciativa de salud para las mujeres (WHI: Women's Health Initiative). No obstante, revisiones del WHI y evidencias actuales determinan que la TH: (a) es la terapia más efectiva de los síntomas vasomotores, de sus potenciales consecuencias (disminución en calidad del sueño, irritabilidad, reducción en calidad de vida), y para tratar la atrofia urogenital. (b) Previene la osteoporosis y en pacientes con endotelios sanos, reduce el riesgo de hipertensión arterial, diabetes II y enfermedad coronaria, siendo el cociente beneficio/ riesgo mayor cuando se inicia tempranamente al sucederse el hipoestrogenismo. (c) Existe la tendencia a utilizar dosis mínimas, estrógenos no orales y progestágenos puros, para disminuir sus riesgos. (d) Tibolona y paroxetina son las mejores alternativas para tratar los síntomas vasomotores. (e) Finalmente, los cambios a estilo de vida saludable son coadyuvantes excelentes de la TH en el manejo de los síntomas menopaúsicos y en la prevención de las enfermedades crónicas de la postmenopausia, las cuales ameritan tratamientos específicos.
\end{abstract}

Palabras clave: menopausia, terapia de reemplazo hormonal, alteraciones sexuales, enfermedades cardiovasculares, osteoporosis, cáncer de mama

\section{MENOPAUSE: AN OVERVIEW OF CURRENT ASSESSMENT AND MANAGEMENT}

\begin{abstract}
There has been a marked decline in hormonal therapy (HT) for menopausal symptoms, since 2002, due the understanding of the benefits and risks as described in the Women's Health Initiative (WHI). Nevertheless, revisions of the WHI and current evidences determine that the HT: (a) is the most effective treatment for menopause vasomotor symptoms and their potential consequences (diminished sleep quality, irritability, and reduced quality of life), and to treat urogenital atrophy. (b) prevents the osteoporosis and, in women with healthy endothelial arteries, it reduces the risk of hypertension, diabetes II and coronary heart disease; but, the benefit-risk ratio is favorable for women who initiate HT close to ovarian failure or menopause. (c) There is the tendency to minimize dose, give no oral estrogens and pure progestagens, in order to diminish its potential risks. (d) Tibolona and Paroxetine are good alternatives to treat menopausal vasomotor symptoms. (e) Finally, the changes in the lifestyle are excellent HT adittional aids to prevent postmenopausal chronic diseases; but these diseases deserve specific treatments.
\end{abstract}

Key words: menopause, hormonal therapy, sexual dysfunctions, cardiovascular diseases, osteoporosis, breast cancer

\section{Menopausa: PANORAma ATUAL DE ABORDAGEM}

\section{Resumo}

Ainda permanece, desde 2002, uma diminuição acentuada no uso de terapia hormonal clássica (TH) para a abordagem da menopausa, devido aos riscos identificados no estudo Iniciativa de saúde para mulheres (WHI: Women's Health Initiative). No entanto, revisiões WHI e as evidências atuais determinam que o TH: (a) é a terapia mais eficaz para os sintomas vasomotores, as suas possíveis conseqüências (diminuição da qualidade de sono, irritabilidade, redução da qualidade

Recibido: Abril 6 de $2011 \quad$ Aceptado: Junio 1 de 2011

* Correspondencia: Luis Ernesto Pérez leperez@cable.net.co. Dirección postal: Tr. 3 No 49-00, Facultad de Medicina, Universidad Militar Nueva Granada, Bogotá, Colombia. Teléfono 315-3322269 
de vida), e tratamento de atrofia urogenital. (b) Previne a osteoporose, e em pacientes com endotélio saudável, reduz o risco de hipertensão, diabetes tipo II e doença arterial coronariana, com a relação risco / benefício maior quando o inicio do hipoestrogenismo é cedo. (c) Há uma tendência a utilizar pequenas doses, de estrógenos não-orais e progestágenos puros, para reduzir seus riscos. (d) Tibolona e paroxetina são as melhores alternativas para o tratamento de sintomas vasomotores. (e) Finalmente, as alterações a estilos de vida saudável são excelentes auxiliares da HT na abordagem dos sintomas da menopausa e prevenção de doenças crônicas na pós-menopausa, que merecem um tratamento específico.

Palavras-chave: menopausa, terapia de reposição hormonal, disfunção sexual, doenças cardiovasculares, osteoporose, câncer de mama

\section{Introducción}

La función ovárica puede perderse, a lo largo de la vida de la mujer, por varias causas: (a) Falla ovárica prematura (amenorrea por patología ovárica antes de los 40 años de edad). (b) Oforectomía médica o quirúrgica. (c) Menopausia prematura (cuando ocurre antes de los 40 años). (d) Finalmente por, menopausia. Ésta, última causa, ocurre en promedio a los 50 años de edad, con amplio rango entre 48 y 58 años, dependiendo de factores como predisposición genética, paridad, índice de masa corporal, nivel educativo, altitud geográfica de la población, tabaquismo y recursos económicos. En Estado Unidos ocurre en promedio a los 51 años, en Latinoamérica a los 49 y en Colombia a los 48 años (Bogotá 48,8 $\pm 5,4$ ) (1).

Esta es una revisión sistemática cualitativa de las publicaciones más relevantes de los últimos diez años encontradas en las principales bases de datos (Cochrane, Ovid, PubMed-MESH, guías de manejo), cuyo objetivo es actualizar, con las mejores evidencias médicas, el manejo de la mujer menopausica.

\section{Fisiopatología}

Varios hechos van marcando, y predicen, la pérdida paulatina de la función ovárica antes de la menopausia: (a) En los últimos años de la vida reproductiva, desde los 36 , el ovario va progresivamente mostrando menor cantidad de folículos antrales y disminuyendo la producción de AMH (hormona antimulleriana), el mejor marcador endocrino de reserva ovárica a esa edad. (b) En los últimos cinco años (premenopausia temprana) cae también la producción de inhibina $\mathrm{B}$, con $\mathrm{FSH}>10 \mathrm{mUI} / \mathrm{mL}$, y estradiol alto paradójicamente; entonces, las mujeres acusan polimenorreas. (c) En la peri-menopausia inicial (dos años previos a la menopausia), además, bajan la inhibína A y estrógenos, FSH entre 10 y $30 \mathrm{mUI} / \mathrm{mL}$, y presencia clínica de oligomenorreas. (d) En la peri-menopausia avanzada (año previo) los estrógenos continúan bajando y la FSH continúa subiendo, con ciclos menstruales mayores de tres meses. (e) Finalmente, en la peri-menopausia avanzada (año siguiente) hay marcado hipoestrogenemia, FSH > $40 \mathrm{mUI} / \mathrm{mL}$, amenorrea mayor de un año y, en definitiva, menopausia $(2,3)$.

Los síntomas vasomotores, y su severidad, se relacionan con la pérdida progresiva de estrógenos endógenos desde la peri-menopausia. A lo largo de la post-menopausia temprana (los primeros 5 años desde la menopausia), además, suelen aparecer en forma progresiva, alteraciones de la libido, cambios de humor, atrofia vulvar, atrofia vaginal, dispareunia, incontinencia urinaria, infección urinaria recurrente y osteopenia. Y durante la post-menopausia tardía (dese los 5 años de la menopausia hasta la muerte) son frecuentes, osteoporosis, fracturas osteoporóticas, enfermedad cardiovascular (ECV), depresión y en algunas mujeres demencia.

\section{Valoración inicial de la paciente menopáusica}

Una valoración integral es imprescindible: (a) Historia clínica enfocada a los síntomas y su severidad, edad y estilos de vida (dieta, cigarrillo, alcohol, ejercicio físico), y antecedentes relevantes personales y familiares (mamarios, ECV, diabetes). (b) Examen físico con énfasis en Tensión Arterial, IMC, circunferencia abdominal, alteraciones mamarias, signos de ECV, osteoporosis, atrofia urogenital e incontinencia urinaria, entre otros. (c) Paraclínicos, acorde a cada paciente, FSH, estradiol, TSH, mamografía (tamizaje entre los 50 y 70 años, o antes si factores de riesgo), perfil lipídico, glicemia y densitometría ósea (tamizaje a partir de los 65 años, o desde la menopausia si factores de riesgo).

\section{Cambios a estilo de vida saludable}

Se debe insistir, con recomendación A en evidencias, que toda paciente menopáusica asuma estilo de vida saludable para disminuir los síntomas de la menopausia, los riesgos de la $\mathrm{TH}, \mathrm{y}$ los riesgos de las enfermedades propias de 
la tercera edad (ECV, diabetes II, hipertensión arterial, CA-mamario, osteoporosis, etc). Este debe incluir: (a) Dieta sin grasas, pero rica en omega 3 que son los ácidos grasos esenciales poliinsaturados, como EPA y DHA (nueces, atún, sierra, bonito, salmón, sardinas), carnes blancas, frutas, verduras, calcio, agua, y cereales ricos en fibra; o la "dieta mediterránea" en la que predominan los alimentos obtenidos de los cultivos tradicionales de esta zona geográfica bañada por el mediterráneo: trigo, olivo, vid, hortalizas, frutas, frutos secos y pescados. (b) Ejercicio físico adecuado, tipo aeróbico moderado, $30 \mathrm{~min} / \mathrm{día}$ 3 veces por semana, para mantener el IMC entre 20 y 25 y el perímetro abdominal menor de $88 \mathrm{~cm}$. (c) Ejercicios mentales y espirituales para mantener la función cognitiva y manejar el estrés. (d) Evitar cigarrillo, bebidas calientes y alcohol, conocidos disparadores de los síntomas vasomotores $(4,5)$.

\section{Síntomas vasomotores}

La disminución de estrógenos induce inestabilidad de la GnRH con incremento de serotonina y de sus receptores hipertérmicos (5HT-2 $\alpha)$, en el centro termorregulador localizado en el área preóptica, que ocasionan oleadas de calor. Por otro lado, las bajas concentraciones de estrógenos también inducen la producción hipotalámica de opiodes, especialmente de $\beta$-endorfinas, y noradrenalina que tienen también injerencia en la desestabilización del centro termorregulador $\mathrm{y}$, adicionalmente, en la regulación del tono vascular periférico ocasionando sudoración nocturna profusa, palpitaciones, ansiedad. Estos síntomas vasomotores y sus potenciales consecuencias (irritabilidad, trastornos del sueño y disminución de calidad de vida) los sufren el $60-80 \%$ de las menopáusicas: perimenopausia inicial $14-51 \%$ y peri-menopausia avanzada y post-menopausia $30-80 \%$.

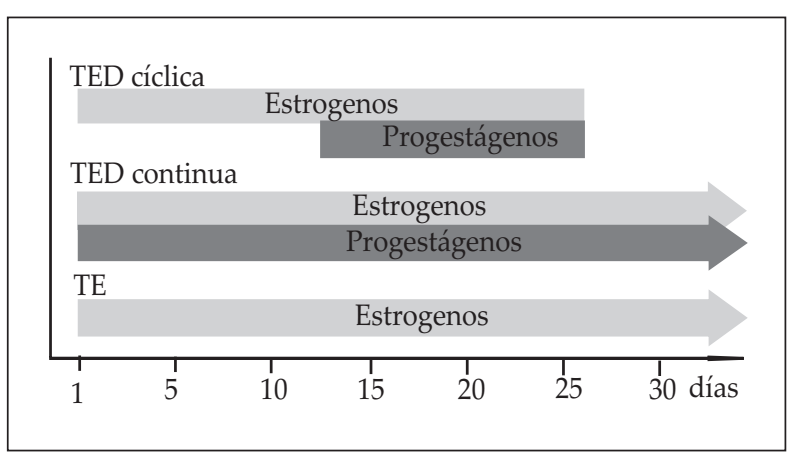

Figura 1. Tres formas frecuentes de terapia hormonal TEP: estrógenos más progestágenos; TE estrógenos.
El tratamiento de los síntomas vasomotores se hace en la actualidad con $\mathrm{TH}$ junto con cambios de estilo de vida como coadyuvantes efectivos, o con terapias alternativas en caso de contraindicaciones.

1. Terapia hormonal clásica. La $\mathrm{TH}$, en sus diferentes formas (Tabla 1), es actualmente el tratamiento más efectivo de los síntomas vasomotores, de moderados a severos y de sus potenciales consecuencias. La terapia con solo estrógenos (TE) para pacientes sin útero y la terapia con estrógenos y progestágenos (TEP) para pacientes con útero, a fin de protegerlo efectivamente de la hiperplasia y carcinoma endometriales $(5,6)$.

Hasta el 2002 se había extendido el uso indiscriminado, con tiempo indefinido, de la TH para toda menopáusica, apoyada en estudios observacionales que le otorgaban multibeneficios. Pero, los resultados del WHI (7), un importante estudio aleatorizado controlado (RCT), pusieron en evidencia como riesgos de la TEP: cáncer mamario, RR 1,29 (1,0-1,59); trombo embolismo pulmonar, RR 2,13 (1,39-3,25); accidente cerebro vascular, RR 1,41 (1,07-1,85) y enfermedad coronaria, RR 1,29 $(1,02-1,63)$. Y más tarde, en el 2004, los resultados del brazo de el WHI (8) con solo estrógenos (TE) evidenciaron su riesgo de accidente cerebro vascular trombótico, RR 1,39 (1,10-1,17) Estos riesgos de la $\mathrm{TH}$ mostrados por el WHI, alarmaron tanto a médicos como a pacientes, de tal suerte que su inmediata y persistente consecuencia, fue el derrumbamiento de la $\mathrm{TH}$ hasta en el $90 \%$.

No obstante, revisiones del estudio WHI, meta-análisis de estudios observacionales y de RCTs, al igual que guías de manejo como la de la sociedad americana de menopausia (NAMS: North American Menopause Society), han demostrado que esos riesgos no se pueden generalizar: Primero, porque en los WHI incluyeron pacientes con edad promedio de 63 años, con muchos tratamientos iniciados hasta diez años después de la menopausia, y sólo utilizaron estrógenos conjugados y medroxiprogeterona orales. En segundo lugar, han mostrado en forma contundente que el cociente beneficio/riesgo de la TH es mayor cuando se inicia tan pronto cuando sucede la falla ovárica prematura, la menopausia prematura, o la menopausia, y va disminuyendo con la edad de la paciente, tornándose negativo a partir de los 60 años, cuando aparecen multiriesgos propios de esa edad. En tercer lugar, han mostrado que en mujeres jóvenes la TH abre "una ventana de oportunidad" para prevenir osteoporosis, propiciar disminución en la aparición de hipertensión arterial, diabetes II y de enfermedad cardiaca coronaria. Y, finalmente, han recalcado que muchos de esos riesgos se relacionan con varios 
factores como, riesgos basales de enfermedades para los cuales la TH está contraindicada, edad, edad de inicio de la menopausia o la falla ovárica, tiempo de postmenopausia sin $\mathrm{TH}$, tipo de hormonas, dosis, ruta de administración, tiempo de duración, y de enfermedades emergentes durante el tratamiento $(5,6,9,10-12)$.

Por supuesto, la TH clásica no se debe administrar cuando está contraindicada: embarazo o lactancia, hemorragia uterina anormal sin diagnóstico, riego o carcinoma mamario, neoplasias influidas por esteroides sexuales, enfermedad hepática, trombo embolismo arterial o venoso, enfermedad coronaria o cerebrovascular, e hipertrigliceridemia $(5,6,9)$.

Consecuentemente, en la actualidad, las evidencias médicas indican que si bien es cierto que el péndulo está regresando de la posición negativa a la positiva de la $\mathrm{TH}$, es conveniente seguir las siguientes recomendaciones para formularla:

a. Tipo de TH: preferir estrógenos por vía trans-cutánea o parenteral (punto controvertido) como parches, gel y ampollas, evitando el primer paso hepático de los orales que aumenta factores pro coagulantes. Igualmente, abandonar el uso de progestinas "impuras" como el acetato de medroxiprogesterona (efectos glucocorticoide y androgénico) o las norderivadas (efectos androgénicos) favoreciendo las puras como dienogest, drosperinona, dihidroprogesterona, o progesterona micronizada, porque son bio-equivalentes pero con menos riesgos $(5,13,14)$.

b. Dosis: formular las mínimas necesarias por día. $17 \beta$ estradiol trans dérmico 14-25 $\mu$ g; estradiol oral 0,5 a $2 \mathrm{mg}$; estrógenos conjugados entre 0,15 a 0,3 mg; progesterona micronizada 50 a $100 \mathrm{mg}$; drosperinona 0,5 a $2 \mathrm{mg}$; medroxiprogesterona 1,5 a $5 \mathrm{mg}$ (si no hay otra opción).

c. Inicio y duración: iniciarla lo más pronto posible ante la falla ovárica prematura, o la menopausia, aprovechando el endotelio normal, como ventana de oportunidad para beneficios adicionales al tratamiento de los síntomas vasomotores. Dejarla continuamente, "y con prudencia" hasta completar la post menopausia temprana; $y$, si los beneficios superan los riesgos, acorde con cada paciente, prologarla en la post menopausia tardía, sin pasar de los 60 años de edad, cuando se elevan los multi riesgos basales de ECV y de CA-mamario, entre otros (15-18).

d. Establecer la necesidad de controles periódicos.

e. Recomendar siempre estilo de vida saludable.
2. Tibolona. Es una de las alternativas, a la $\mathrm{TH}$, para tratar los síntomas vasomotores en la postmenopausia temprana $(2,5 \mathrm{mg} / \mathrm{dí})$. Especialmente útil en pacientes, que además, presentan pérdida de la libido, resequedad vaginal $\mathrm{y}$ trastornos del ánimo. Sus metabolitos $3 \alpha-\mathrm{OH}$ (3-alfa-hidroxi) y $3 \beta-\mathrm{OH}$ (3-beta-hidroxi) tienen propiedades estrogénicas y la $\Delta 4$ (isómero delta 4) propiedades progestacional y androgénicas. Disminuye SHBG, triglicéridos HDL y LDL del colesterol. Aumenta testosterona libre y allopregnenolona (sedante) (19).

Sus acciones y efectos colaterales han sido ampliamente documentados con múltiples estudios observacionales, RCTs y meta-análisis (LISA, LIFT, OPAL, THEBES, GPRD, LIFT, Millón de mujeres, LIBERATE) $(20,21)$. Sus resultados permiten concluir: (a) Es efectiva en el tratamiento de los síntomas vasomotores, resequedad vaginal, alteraciones del ánimo y pérdida de la libido. (b) Aumenta la densidad mineral ósea y disminuye significativa el riesgo de fracturas. (c) Es antilipemiante y disminuye el riesgo coronario durante la postmenopausia temprana, pero no en la tardía, en comparación con el placebo. (d) Es protectora del endometrio produciendo atrofia, aunque, inicialmente puede producir manchados. (e) Es ligeramente protectora de la mama, reduciendo el riesgo de carcinoma invasor, pero, con riesgo mayor de recurrencia. (f) Pero, también está contraindicada, como la $\mathrm{TH}$, en carcinoma mamario y trombo-embolismo.

3. Inhibidores de la recaptación de serotonina. Constituyen la opción terapéutica cuando tanto $\mathrm{TH}$ como Tibolona están contraindicados. Ellos corrigen la alteración serotoninérgica ocasionada por la baja de estrógenos y han mostrado disminución significativa de los síntomas vasomotores respecto al placebo, en pacientes supervivientes de CA mamario. Paroxetina 10-20 mg/día reduce los síntomas vasomotores en el 67-75\% y Fluoxetina $20 \mathrm{mg} /$ día solo en el $42 \%$ (22).

4. Fitoestrógenos. Solamente útiles para pacientes con síntomas leves ya que su efectividad es parecida al placebo o ligeramente superior, pero sin diferencia significativa. El meta-análisis de Cochrane (30 RCTs/ 2007) mostró que los fitoestrógenos no superan, significativamente, al palcebo (23). Una revisión sistemática (23 RCTs/2009) determinó un ligero beneficio, no significativo, sobre el placebo (24) y en otro meta-análisis (19 RCTs/2010) en donde se comparó el placebo contra suplementos dietéticos y extractos de soya, tabletas de genisteina o daidzeina, no se obtuvieron resultados definitivos, debido a la heterogeneidad de los estudios (25). 


\section{Síntomas genitourinarios y sexuales}

La falta de estrógenos produce atrofia de la mucosa vaginal, de la uretral, del prepucio del clítoris, del colágeno y del tejido adiposo vulvar, con disminución de los lubricantes glandulares. Ocurre resequedad vaginal, prurito, incontinencia urinaria, infección urinaria recurrente, dispareunia y, en parte, pérdida de la libido. La TH, sistémica para síntomas vasomotores, es también la terapia para los síntomas genitourinarios. Sin embargo, cuando se dan bajas dosis de estrógenos, a veces, no es suficiente y es necesario agregar estrógenos locales: cremas, anillos intravaginales de liberación sostenida o tabletas intravaginales. Precisamente, la terapia local es la que se debe formular cuando estos síntomas se presentan aisladamente $(26,27)$.

La incontinencia urinaria se incrementa con la edad. Los estrógenos locales la disminuyen, cuando hay atrofia. Por consiguiente, antes de la intervención quirúrgica, se debe estimular el uso de estrógenos locales, cambios del estilo de vida, ejercicios vesicales de Kegel, estimulación eléctrica funcional, y antimuscarínicos (28,29). La TH sistémica alivia la dispareunia, pero mejor, las cremas de estrógenos. Cuando la libido es el problema, la Tibolona o los andrógenos transdérmicos ayudan mucho. Aunque, los problemas sexuales ameritan un abordaje psico-bio-sociosexual bajo contextos de cambios físicos, intrapersonales, interpersonales y sociales $(30,31)$.

\section{Cambios de ánimo y depresión}

Los mecanismos que lo producen no son muy claros. Se ha sugerido que es resultado de antecedentes depresivos, déficit de estrógenos, síntomas vasomotores, alteraciones del sueño, síntomas molestos propios de la edad avanzada, cambios en la imagen corporal y alteraciones de la función sexual (32). Al respecto, los efectos de la TH son controvertidos: un RCT no le da beneficios y otros dos la ponen como coadyuvantes previa a psicoterapia o antidepresivos inhibidores de la recaptación de serotonina (33).

\section{Alteraciones cognitivas y enfermedad de Alzheimer}

Los estrógenos favorecen las sinapsis neuronales y la proliferación de sus espinas dendríticas, por lo cual se piensa que las afectaciones de la memoria episódica y de la producción verbal de las menopaúsicas, son causadas por el déficit de sus estrógenos. Estudios de cohorte con seguimientos hasta por 27 años post oforectomía bilateral han mostrado ese riesgo en pacientes sin $\mathrm{TH}$ en comparación con menopáusicas jóvenes tratadas $(34,35)$. Pero, la TH en menopausia tardía ha mostrado efectos adversos, según se desprende de un meta-análisis (36 RCTs/2020) (36); y en mujeres de 65 y más años, incluso, la TH puede producir disminución del volumen del hipocampo $(\mathrm{P}=0,05)$, lóbulo frontal $(\mathrm{P}=0,004)$ y cerebro total $(\mathrm{P}=0,07)$ (37). Por otro lado, los estudios demuestran que los ejercicios de memoria y concentración son la mejor profilaxis de las alteraciones cognitivas (38).

La Enfermedad de Alzheimer tiene fundamentalmente bases genéticas y su fisiopatología es compleja y global afectando neuronas y glía. En un meta-análisis realizado por el Instituto Nacional de Salud de Estados Unidos, con 300 estudios observacionales y 50 RCTs, contundentemente no encontraron causas ambientales, dietéticas ni farmacológicas de la enfermedad de Alzheimer; y también observaron que no había diferencia entre el placebo y las diversas terapias que se han utilizado (dietas, vitaminas, ginkgo biloba, medicamentos, estrógenos, etc) (39).

\section{Enfermedad cardiovascular y terapia hormonal}

Respecto a ECV, nos referiremos a enfermedad coronaria (EC), trombo-embolismo venoso (TEV) y accidente cerebro-vascular (ACV). Las mujeres, a diferencias de los hombres, desde la vida intrauterina tienen sus endotelios arteriales densamente poblados de receptores estrogénicos $\alpha$ y $\beta$, para que los estrógenos ejerzan funciones protectoras en ellos (40). Desde el punto de vista genómico, los estrógenos aumentan la síntesis y concentración de óxido nítrico y disminuyen los depósitos de calcio endoteliales favoreciendo la dilatación y disminuyendo la arterioesclerosis. Estimulan, además, la vasodilatación al disminuir las acciones de la renina y de la enzima convertidora de angiotensina I con disminución en la formación de angiotensina II. También disminuyen las posibilidades de formación de placas ateromatosas al favorecer mejores concentraciones de HDL del colesterol. Además, son inhibidores de la mitosis del músculo liso de las paredes arteriales pero no de las células endoteliales favoreciendo los mecanismos de revascularización. Y, desde el punto de vista no genómico, en forma aguda son vasodilatadores aumentando el óxido nítrico, antiinflamatorios y protectores de los cardiomiocitos. Sin embargo, los mecanismos benéficos de los estrógenos son ejercidos en endotelios sanos, porque los estudios han demostrado que en endotelios alterados por hipertensión, diabetes, obesidad o envejecimiento se minimizan o son contrarios $(2,11,13)$.

En las décadas de los 80 y 90, las acciones benéficas de los estrógenos, sobre la función arterial, fueron ampliamente documentadas en estudios observacionales recalcando que, dichos beneficios de género, caían abruptamente en 
las mujeres con la llegada de la menopausia poniéndolas en riesgos aún mayores que los hombres de morbilidad y mortalidad por ECV. Por lo tanto, la TH se recomendaba con entusiasmo para protección prolongada de la función cardiovascular. Pero, los estudios HERS (Heart and Estrogen/progestin Replacement Study) (41) y WHI $(7,8)$ derrumbaron la $\mathrm{TH}$, al no encontrar beneficio, pero si sus conocidos riesgos.

Por otro lado, en los últimos nueve años, tanto estudios observacionales como RCTs, incluyendo revisiones del WHI originales y otra rama del WHI con pacientes de 50 a 59 años, han ido rescatando los beneficios cardiovasculares de los estrógenos, especialmente como protectores de enfermedad coronaria, hipertensión arterial, y desde minimización hasta neutralización del riesgo para TEV y $\mathrm{ACV}$. De hecho, esos beneficios son notorios si se administran tempranamente una vez suceda el hipoestrogenismo, por cualquier causa, aprovechando endotelios sanos como "ventana de oportunidad" que se continúa hasta los 60 años de edad, cuando se dan estrógenos no orales, evitando el primer paso hepático, y progestágenos puros, ambos, con las dosis mínimamente efectivas. Contrariamente, cuando la TH se inicia años después de ocurrida la menopausia, en menopáusicas mayores, o con desarrollo de arterioesclerosis, los estrógenos causan inestabilidad y ruptura de la placa arterioesclerótica ocasionando trombosis e infarto $(12,42-47)$.

Desde luego, ningún estudio recomienda la $\mathrm{TH}$ como prevención primaria de la ECV, a ninguna edad. Pero, los efectos benéficos cardiovasculares se aprovechan cuando se da para tratar síntomas vasomotores y atrofia urogenital, tempranamente desde el inicio del hipoestrogenismo y hasta los 55 años de edad y se continúa con prudentes controles hasta los 60. Sin olvidar, que a las mujeres con riesgos de ECV (obesidad, trombofilias, S. metabólico, $>60$ años, etc) se les debe contraindicar la TH y remitirlas al especialista, para estratificar el riesgo (score de Framingham) y ofrecer tratamiento oportuno (47).

Los cambios a estilo de vida saludable sí tienen recomendación $\mathrm{A}$ en evidencias para disminuir los riesgos de ECV, porque con ello se estimula TA $<140 / 90$, IMC 20-25, diámetro abdominal $<88 \mathrm{~cm}, \mathrm{C}-\mathrm{LDL}<100 \mathrm{mg} / \mathrm{dl}$, C-HDL $>50 \mathrm{mg} / \mathrm{dl}$, y $\mathrm{TG}<150 \mathrm{mg} / \mathrm{dl}(11,12)$.

\section{Diabetes mellitus II y terapia hormonal}

Tanto, meta-análisis como RCTs aislados, incluido el WHI, han concluido que la TH disminuye la aparición de diabetes II y beneficia su tratamiento con menores dosis de hipoglucemiantes. Los estrógenos disminuyen la resis- tencia a la insulina y favorecen la expresión de GLUT-4. Sin embargo, la TH no debe ser considerada como terapia $(12,48)$.

\section{Terapia hormonal y riesgos de cáncer}

1. Cáncer de mama. El diagnóstico de Ca de seno se incrementa en pacientes con $\mathrm{TH}$, después de tres cinco años de uso, como ha sido confirmado por el WHI, otros RCTs y por meta-análisis, posiblemente actuando como promotora de lesiones cancerígenas preexistentes y no como factor iniciador $(12,49)$. Los estudios muestran que: (a) La TH está contraindicada en pacientes con riesgo de CA-mamario. (b) La TE y, con mayor impacto, la TEP incrementan la proliferación celular, el dolor mamario y la densidad mamaria, dificultando la interpretación de la mamografía. (c) La TE tiene menos riesgo que la TEP, después de un promedio de 1-7 años de uso, pero no después de 10-15. (d) El riesgo de la TH disminuye (no conformado con RCTs) cuando se utilizan estrógenos transdérmicos y progesterona micronizada. (e) Se ponderan como preventivos los cambios a estilo de vida saludable (RCTs), el autoexamen y el tamizaje ecográfico, con mejor impacto beneficio/riesgo, a partir de los 50 años de edad, cada dos años hasta los 74 y desde antes si hay riesgos; aunque, el National Cancer Institute en USA recomienda el tamizaje a partir de los 40 años de edad (49).

2. Cáncer de ovario. Su asociación con TH es contradictoria. Estudios observacionales le dan cierta asociación y riesgo, cuando se usan por más de cinco años $(9,10,11)$. Sin embargo, el realizado por el WHI, con un seguimiento por 5,6 años, no mostró diferencias significativas respecto al placebo (12).

3. Cáncer de endometrio. El riesgo no se incrementa con TEP, pero si con ET. Consecuentemente, los progestágenos están indicados con el exclusivo propósito de evitar éste riesgo. Sin embargo, la TH no está indicada en pacientes con historia de Ca endometrial $(9,10,11)$.

4. Cáncer de colon. No se incrementa el riesgo con TH (45).

\section{Osteoporosis post menopáusica}

Desde los 35 años se inicia el proceso de remodelación ósea y desde entonces se pierde $0,5-1 \%$ por año. Durante toda la vida los estrógenos protegen el hueso: estimulan la formación de osteoblastos por medio del TGF-b (Factor de crecimiento tumoral beta); inhiben las citoquinas reclutadoras de osteoclastos (IL-1, IL-6, TNFa y GM-CSF, RANKL); y contrarrestan los mecanismos de resorción ósea y la movilización del calcio inducida por PTH. La 
progesterona, por su parte, estimula los osteoblastos a depositar matriz mineralizada.

La osteoporosis con riesgo de fracturas, se produce 10-15 años después de la menopausia natural o inducida. Se diagnostica con densitometría ósea, realizando tamizaje a partir de los 65 años, o desde la menopausia para obtener beneficios diagnósticos y terapéuticos. Y se previene con cambios en estilo de vida saludable, protección externa, calcio, vitamina $\mathrm{D}, \mathrm{TH}$, e incluso con raloxifen y bifosfonatos en pacientes con alto riesgo (Tabla 1 ).

1. Calcio. Su ingesta excesiva, en mujeres sanas, se ha asociado a infartos, ACV y muerte repentina (RCT/2008, Ca vs placebo, $n=1471$ ) (50). Se debe tener en cuenta el cociente beneficio/riesgo y seguir la sugerencia de la OMS de dar dosis de $500 \mathrm{mg} /$ día, o tan solo dieta rica en calcio.

2. Vitamina D. Los niveles fisiológicos son deficientes a partir de los 65 años de edad, a pesar de dieta rica en vitamina $\mathrm{D}$ y buen sol. Es útil medir la $25(\mathrm{OH}) \mathrm{D}$ plasmática (Normal > $20 \mathrm{ng} / \mathrm{mL}$ ) y dar una dosis de vitamina D de 800 a $1000 \mathrm{UI} /$ día, que generalmente la mantiene por encima de esos niveles (51).

3. Terapia hormonal. En la actualidad no existe duda, según RCTs, que la TH es el mejor manejo profiláctico de la osteoporosis, especialmente en pacientes con falla ovárica prematura y menopausia prematura: en diez años reduce el riesgo de fracturas en un $50 \%(R R=0,5)$ mientras que en dos años solo lo hace en el $20 \%$ (RR
$=0,8)$. TH debería ser la primera línea de tratamiento, pero no ha sido aprobada como tal $(8,12,50,52,53)$.

Ya, para el tratamiento de la osteoporosis existen actualmente varias opciones: (Tabla 1 ).

1. Raloxifén. Es un modulador selectivo del receptor de estrógenos (SERM) con acción agonista en hueso y antagonista en glándula mamaria usado en pacientes con alto riesgo o con cáncer mamario $(55,56)$.

2. Bifosfonatos. Respeto a ellos, se ha establecido la longitud temporal para administrarlos, a partir de la cual se puede descansar y revalorar para continuarlos o no, más tarde: Alendronato se puede utilizar por diez años, dadas sus buenas acciones en vértebras y cadera; Risedronato, por cinco años; Ibandronato una dosis oral mensual, o una dosis I.V trimestral, durante tres años; y Zolendronato I.V. cada año, por tres años. Téngase en cuenta que con los bifosfonatos orales, dados por mucho tiempo, se corre el riesgo de microdaños, fragilidad ósea, fracturas atípicas, y con los I.V. el riesgo de osteonecrosis de mandíbula $(57,58)$.

3. Teriparatide (PTH). Muy útil, por 18 a 24 meses, en pacientes con osteoporosis grave y antecedente o alto riesgo de fracturas $(59,60)$.

4. Ranelato de estroncio. Tiene acción dual al estimular los osteoblastos e inhibir los osteoclastos, pero con poca adherencia por necesitarse una dosis oral diaria y presentar alguans reacciones adversas gastrointestinales (61).

Tabla 1. Prevención y Tratamiento de la osteoporosis Post Menopáusica.

\begin{tabular}{|c|c|c|c|c|}
\hline Manejo & Agente & Dosis & Via & Reducción fracturas \% V/C \\
\hline \multirow{6}{*}{ Preventivo } & Protectores externos & & & 160 \\
\hline & $\mathrm{Ce}$ & $500 \mathrm{mg} / \mathrm{d}$ & Oral & \\
\hline & Vit. D & $500-1000 \mathrm{UI} / \mathrm{d}$ & Oral & \\
\hline & Calcitriol & $0,25 \mathrm{Ug} / \mathrm{d}$ & Oral & \\
\hline & Estrógenos & $0.625-1.23$ & TD-IM-Vg & $50 \%$ \\
\hline & Tibolona (Livial) & $2,5 \mathrm{mg} / \mathrm{d}$ & Oral & $45 / 36$ \\
\hline \multirow{9}{*}{ Tratamiento } & Raloxifen (SERM) & $60 \mathrm{mg} / \mathrm{d}$ & Oral & $50 / 10$ \\
\hline & Alendronato (Fosamax) & $70 \mathrm{mg} / \mathrm{s}$ & Oral & $48 / 55$ \\
\hline & Risedronato (Actonel) & $5 \mathrm{mg} / \mathrm{d}, 35 \mathrm{mg} / \mathrm{s}$ & Oral & $41 / 39$ \\
\hline & Ibandronato (Bonviva) & $150 / m-3 / 3 m$ & Oral / IV & $55 / 35$ \\
\hline & Zolendronato (Aclasta) & $5 \mathrm{mg} / \mathrm{año}$ & IV & $70-41$ \\
\hline & PTH (Teriparatide) (forteo) & $20 \mathrm{Ug} / \mathrm{d}$ & SC & NF $-65 / 53$ \\
\hline & Calcitonina & $200 \mathrm{UI} / \mathrm{d}$ & IN-SC-IM & $21 \%$ \\
\hline & Ranelato de Sr (Protos) & $2 \mathrm{~g} / \mathrm{d}$ papeletas & Oral & $49 / 41$ \\
\hline & Denosumbab & $60 \mathrm{mg} / \mathrm{cada} 6 \mathrm{~m}$ & SC & $68 / 20$ \\
\hline
\end{tabular}

V/C, Vertebral; TD, transdérmico; IV, intravenoso; SC, subcutánea; NF, nuevas fracturas. 
5. Calcitonina. En la actualidad solo útil como analgésico en fracturas vertebrales, dado que un metaanálisis (RCTs/20029) no mostró diferencia significativa en reducción del riesgo de fracturas con respecto al placebo o calcio-Vit D (62).

6. Denosumab. Es un anticuerpo monoclonal que inhibe la formación y función de los osteoclastos al unirse al RANKL e impedir la interacción con su receptor el RANK, previniendo así la resorción y desmineralización ósea. Parece la terapia del futuro porque, a dosis de $60 \mathrm{mg}$ S.C. cada seis meses, disminuye el riesgo de fracturas de cadera, vertebrales y no vertebrales, y en el estudio en el que se demostró su acción se observó que las reacciones colaterales presentadas eran iguales a las inducidas por el placebo (63).

\section{Conclusiones}

Sin lugar a duda, la mujer menopáusica amerita un manejo integral en favor de una buena calidad de vida. Este enfoque debe incluir: TH para los síntomas menopáusicos, tratamiento específicos para las enfermedades crónicas, y por supuesto, estilos de vida saludables con dieta y ejercicio físico y mental como coadyuvantes fundamentales en la prevención de las enfermedades propias de la tercera edad.

Actualmente, la TH está siendo rescatada de la caída que sufrió con el WHI. Se está puntualizando que, su cociente beneficio/riesgo es máximo en el momento en que sucede la deficiencia estrogénica, bien sea al llegar la menopausia o por falla gonadal prematura (iatrogénica o patológica) y va disminuyendo con el progreso de la edad, invirtiéndose a partir de los 60 años, cuando aparecen otros factores de riesgo. Por su puesto, está indicada, si los beneficios superen los riesgos, para tratar los síntomas vasomotores y atrofia urogenital, con el agregado potencial de mejorar calidad del sueño, libido, función sexual y calidad de vida. Adicionalmente, cuando se da con la indicación anterior, y especialmente cuando la deficiencia estrogénica ocurre tempranamente en la vida de la mujer, le abre una "ventada de oportunidad" para prevenir especialmente osteoporosisfracturas, pero también, diabetes II, hipertensión arterial y enfermedad coronaria, en pacientes con endotelios hasta entonces sanos. Sin embargo, con el objetivo de disminuir los riesgos de la $\mathrm{TH}$, está apareciendo la tendencia de inicio temprano, utilización de mínimas dosis terapéuticas, con estrógenos no orales y progestágenos con perfil metabólico puro. Y como mejores alternativas a la $\mathrm{TH}$, para síntomas vasomotores, están Tibolona o Paroxetina.
Por último, se enfatiza que las enfermedades crónicas de la postmenopausia (ECV, diabetes II, osteoporosis, E. de Alzheimer) se deben manejar con tratamientos específicos, generalmente por médicos especializados.

\section{Referencias}

1. Gómez G. Menopausia: Una visión Global. En: Pérez L E. Infertilidad y Endocrinología Reproductiva. Tercera edición basada en evidencias. Barranquilla - Colombia. Grafimpresos Donado; 2007. p 423-33.

2. Brantes S. Perimenopausia. En: Climaterio y Menopausia en el siglo XXI. Santiago de Chile. Imprenta Naval LTDA; 2010. p 22-28.

3. Van Rooij I, Broekmans F, Scheffer G. et al. Serum antimullerian Hormone levels best reflect the reproductive decline with age in normal women with proven fertility: a longitudinal study. Fertil Steril. 2005;83:979-87.

4. Daley A, Stokes-Lampard H, Macarthur C. Exercise for vasomotor menopausal symptoms. Cochrane Database Syst Rev. 2011;11; 5:CD006108.

5. Holloway D. An overview of the menopause: assessment and management. Nurs Stand. 2011;5;25(30):47-57.

6. Arteaga Urzua E. Terapia de reemplazo hormonal clásica. En: Climaterio y Menopausia en el siglo XXI. Santiago de Chile. Imprenta Naval LTDA; 2010.p 241-9.

7. The Women's Health Initiative Steering Committee. Benefits of estrogen plus progestin in healthy postmenopausal women. Principal results from the Women's health Initiative randomized controlled trial. JAMA. 2002; 288:321-33.

8. The Women's Health Initiative Steering Committee. Effects of conjugated equine estrogens in postmenopausal women with hysterectomy: The Women's Health Initiative randomized controlled trial. JAMA. 2004;291:1701-1.

9. The North American Menopause Society (NAMS). Estrogen and progestogen use in peri-and postmenopausal women: march 2007 position statement of the North American Menopause society. Menopause. 2007;14:1-17.

10. The North American Menopause Society (NAMS). Estrogen and progestagen use in postmenopausal women: position statement 2008. Menopause. 2008;15(4):584-603.

11. The North American Menopause Society (NAMS). Estrogen and progestogen use in postmenopausal women: 2010 position statement of the North American Menopause society. Menopause. 2010;17:242-55.

12. The society of Obstetricians and Gynaecologist of Canada. Menopausia and osteoporosis: Vasomotor symptoms, update 2009. JOGC. 2009;1(S9).

13. Cuadros J L, Fernández-Alonso A M, Chedraui $\mathrm{P}$, et al. Metabolic and hormonal parameters in post-menopausal women 10 years after transdermal oestradiol treatment, alone or combined to micronized oral progesterone. Gynecol Endocrinol. 2011;27(3):156-62.

14. Wang P H, Horng H C, Cheng M H, Chao H T, Chao K C. Standard and low-dose hormone therapy for postmenopausal women--focus on the breast. Taiwan J Obstet Gynecol. 2007;46(2):127-34.

15. Harman S M, Brinton E A, Cedars M, et al. KEEPS: The Kronos Early Estrogen Prevention Study. Climacteric. 2005 Mar;8(1):3-12. 
16. Rossouw J E, Prentice R L, Manson J E, et al. Postmenopausal hormone therapy and risk of cardiovascular disease by age and years since menopause. JAMA. 2007;297(13):1465-77.

17. Hodis H N, Mack W J. Coronary heart disease and hormone replacement therapy after the menopause. Climacteric. 2009;12(1):71-5.

18. Manson J E, Allison M A, Rossouw J E, et al. Estrogen therapy and coronary-artery calcification. N Engl J Med. 2007;356(25):2591-602

19. Hofling M, Carlstrom K, Svane G, et al. Different effects of tibolone and continuous combined estrogen plus progestogen hormone therapy on sex hormone binding globulin and free testosterone levels - an association with mammographic density. Gynecol Endocrinol. 2005:20(2):110-15

20. Segovia A. Tibolona. En: Climaterio y Menopausia en el siglo XXI. Santiago de Chile. Imprenta Naval LTDA; 2010. p 250-256.

21. Archer D F. Endometrium and breast endpoints study (THEBES). RCT multicenter. Fertil Steril. 2007;88(4):866-78

22. Broutin G. Terapia no hormonal para el manejo del síndrome climatérico. En: Climaterio y Menopausia en el siglo XXI. Santiago de Chile. Imprenta Naval LTDA; 2010.p 268-72.

23. Lethaby AE, Brown J, Marjoribanks J, Kronenberg F, Roberts H, Eden J. Phytoestrogens for vasomotor menopausal symptoms. Cochrane Database Syst Rev. 2007;(4):CD001395.

24. Jacobs A, Wegewitz U, Sommerfeld C, Grossklaus R, Lampen A. Efficacy of isoflavones in relieving vasomotor menopausal symptoms - A systematic review. Mol Nutr Food Res. 2009;53(9):1084-97.

25. Bolaños R, Del Castillo A, Francia J. Soy isoflavones versus placebo in the treatment of climacteric vasomotor symptoms: systematic review and meta-analysis. Menopause. 2010;17(3):660-6.

26. Pastore L M, Carter R A, Hulka B S, Wells E. Self-reported urogenital symptoms in postmenopausal women: Women's Health Initiative. Maturitas. 2004;49:292-303.

27. Society of Obstetricians and Gynecologists of Canada. The detection and management of vaginal atrophy. Int J Gynaecol Obstet. 2005;88:222-8.

28. Subak L L, Brown J S, Kraus S R, Brubaker L, Lin F, Richter H E, et al; Diagnostic Aspects of Incontinence Study Group. The "costs" of urinary incontinence for women. Obstet Gynecol. 2006;107:908-16.

29. The society of Obstetricians and Gynaecologist of Canada. Menopausia and osteoporosis: Urogenital health. Update. 2009. JOGC. 2009;1(S27).

30. Graziottin A. Menopause and sexuality: key issues in premature menopause and beyond. Ann N Y Acad Sci. 2010;1205:254-61.

31. Nappi R E, Lachowsky M. Menopause and sexuality: prevalence of symptoms and impact on quality of life. Maturitas. 2009;63(2):138-41.

32. Bromberger J, Matthews K, Schott LL, Brockwell S, Avis NE, Kravitz $\mathrm{HM}$, et al. Depressive symptoms during the menopausal transition: the Study of Women's Health Across the Nation (SWAN). J Affect Disord. 2007;103:267-72.

33. Woods N F, Smith-Dijulio K, Percival D B, Tao E Y, Mariella A, Mitchell ES. Depressed moo d during the menopausal transition and early postmenopause: observations from the Seattle Midlife Women's Health study. Menopause. 2008;15:223-32.

34. Sherwin B B, Henry J F. Brain aging modulates the neuroprotective effects of estrogen on selective aspects of cognition in women: A critical review. Front Neuroendocrinol. 2008;29(1):88-113.
35. Almeida O P, Lautenschlager N, Vasikaram S, Leedman P, Flicker L. Association between physiological serum concentration of estrogen and the mental health of community-dwelling postmenopausal women age 70 years and over. Am J Geriatr Psychiatry. 2005;13(2):142-9.

36. Hogervorst E, Bandelow S. Sex steroids to maintain cognitive function in women after the menopause: meta-analyses of treatment trials. Maturitas. 2010;66(1):56-71.

37. Coker L H, Espeland M A, Rapp S R, Legault C, Resnick S M, Hogan $\mathrm{P}$ et al. Postmenopausal hormone therapy and cognitive outcomes: the Women's Health Initiative Memory Study (WHIMS). J Steroid Biochem Mol Biol. 2010;118(4-5):304-1.

38. The society of Obstetricians and Gynaecologist of Canada. Menopausia and osteoporosis: Mood, memory and cognition. Update 2009. JOGC 2009;1(S31).

39. Daviglus M L, Bell C C, Berrittini W et al. Stete of the science. Conference statement: preventing Alzheimer disease and cognitive decline. Ann Int Med. 2010;153(3):176-81.

40. Melgarejo E. El problema riesgo cardiovascular en la mujer: la ignorancia que mata. Epidemiología, fisiopatología. En, Asociación colombiana de menopausia. Taller salud cardiovascular en el climaterio. Bogotá. Editorial Guadalupe; 2010. p 11-14.

41. Hulley S, Grady D, Bush T, et al. Randomized trial of estrogen plus progestin for secondary prevention of coronary heart disease in postmenopausal women. Heart and Estrogen/progestin Replacement Study (HERS) Research Group. JAMA. 1998;280(7):605-13.

42. Allison M A, Manson J E, Langer R D et al. Oophorectomy, hormone therapy and subclinical coronary artery disease in women with hysterectomy; the WHI coronary artery calcium study. Menopause. 2008;15:639-47.

43. Stevenson J C, Nodis H N, Pickar J H et al. Coronary heart disease and menopause management: The swinging pendulum of HRT. Atherosclerosis. 2009;207:336-40.

44. Harman S M, Vittinghoff E, Brinton E A et al. Timing and duration of menopausal hormone treatment may affect cardiovascular outcomes. Am J Med. 2011;124(3):199-205.

45. LaCroix A Z, Chlebowski R T, Manson J E et al. Health outcomes after stopping conjugated equine estrogens among postmenopausal women with prior hysterectomy: A randomized controlled trial. JAMA. 2011;305(13):1305-14.

46. Salpeter S R, Cheng J, Thabane L, Buckley NS, Salpeter EE. Bayesian meta-analysis of hormone therapy and mortality in younger postmenopausal women. Am J Med. 2009;122(11):1016-22.

47. The society of Obstetricians and Gynaecologist of Canada. Menopausia: Cardiovascular disease. Update 2009. JOGC. 2009;1(S11).

48. Pollak F. Alteraciones del metabolismo de los hidratos de carbono en la menopausia. En: Climaterio y Menopausia en el siglo XXI. Santiago de Chile. Imprenta Naval LTDA; 2010.p. 137-43.

49. Chlebowski R T, Anderson G L, Gass M, et al. Estrogen plus progestin and breast cancer incidence and mortality in postmenopausal women. JAMA. 2010;304(15):1684-92.

50. Bolland M J, Barber P A, Doughty R N, Mason B, Horne A, Ames R et al. Vascular events in healthy older women receiving calcium supplementation: randomised controlled trial. BMJ. 2008;336(7638):262-6.

51. Agarwal S, Agarwal N, Arya S C. Hypovitaminosis D3 in menopausal women in a tertiary care hospital in Delhi. Women's Health. 2011;7(5):607-10. 
52. Society of Obstetricians and Gynecologists of Canada. Menopause and osteoporosis update 2009. Journal of Obstetrics and Gynecology. 2009;2:27-29.

53. Khosla S. Update on estrogens and the skeleton. J Clin Endocrinol Metab. 2010;95(8):3569-77.

54. Britto R, Araújo L, Barbosa I, Silva L, Rocha S, Valente A P. Hormonal therapy with estradiol and testosterone implants: bone protection? Gynecol Endocrinol. 2011;27(2):96-100.

55. Lee W L, Chao H T, Cheng M H, Wang P H. Rationale for using raloxifene to prevent both osteoporosis and breast cancer in postmenopausal women. Maturitas. 2008;60(2):92-107.

56. Messalli E M, Scaffa C. Long-term safety and efficacy of raloxifene in the prevention and treatment of postmenopausal osteoporosis: an update. Int J Women Health. 2010;1:11-20.

57. Delmas P D, Adami S, Strugala C et al. Intravenous ibandronate injections in postmenopausal women with osteoporosis: one-year results from the dosing intravenous administration study. Arthritis Rheum. 2006;54:1838-46.
58. Deeks E D, Perry C M. Zoledronic acid: a review of its use in the treatment of osteoporosis. Drugs Aging. 2008;25(11):963-86.

59. Finkelstein J S, Wyland J J, Lee H, Neer R M. Effects of teriparatide, alendronate, or both in women with postmenopausal osteoporosis. J Clin Endocrinol Metab. 2010 Apr; 95(4):1838-45.

60. Lyritis G P, Georgoulas T, Zafeiris C P. Bone anabolic versus bone anticatabolic treatment of postmenopausal osteoporosis. Ann N Y Acad Sci. 2010;1205:277-83.

61. Deek E D, Dhillon S. Strontium ranelate: A review of its use in the treatment of postmenopausal osteoporosis. Drugs. 2010;70(6):73359 .

62. Cranney A, Tugwell P, Zytaruk N, et al. Meta-analyses of therapies for postmenopausal osteoporosis. VI. Meta-analysis of calcitonin for the treatment of postmenopausal osteoporosis. Endocr Rev. 2002;23(4):540-51.

63. Bogado C E, Boailchuk J A, Zanchetta M B, Massari F E, Zanchetta J R. Denosumab: An update. Drugs Today. 2011;47(8):605-13. 